\title{
Brain structural abnormalities in behavior therapy-resistant obsessive-compulsive disorder revealed by voxel-based morphometry
}

This article was published in the following Dove Press journal:

Neuropsychiatric Disease and Treatment

20 October 2014

Number of times this article has been viewed

\author{
Nobuhiko Hashimoto' \\ Shutaro Nakaaki \\ Akiko Kawaguchi' \\ Junko Sato' \\ Harumasa Kasai ${ }^{3}$ \\ Takashi Nakamae ${ }^{4}$ \\ Jin Narumoto ${ }^{4}$ \\ Jun Miyata ${ }^{5}$ \\ Toshi A Furukawa ${ }^{6,7}$ \\ Masaru Mimura ${ }^{2}$ \\ 'Department of Psychiatry \\ and Cognitive-Behavioral Medicine, \\ Nagoya City University Graduate \\ School of Medical Sciences, \\ Nagoya, Japan; ${ }^{2}$ Department of \\ Neuropsychiatry, Keio University \\ School of Medicine, Tokyo, Japan; \\ ${ }^{3}$ Department of Central Radiology, \\ Nagoya City University Hospital, \\ Nagoya, Japan; ${ }^{4}$ Department \\ of Psychiatry, Graduate School \\ of Medical Science, Kyoto Prefectural \\ University of Medicine, Kyoto, Japan; \\ ${ }^{5}$ Department of Psychiatry, Graduate \\ School of Medicine, Kyoto University, \\ Kyoto, Japan; ${ }^{6}$ Department of Health \\ Promotion and Human Behavior, \\ ${ }^{7}$ Department of Clinical Epidemiology, \\ Kyoto University Graduate School \\ of Medicine/School of Public Health, \\ Kyoto, Japan
}

Correspondence: Shutaro Nakaaki Department of Neuropsychiatry,

Keio University School of Medicine,

35 Shinanomachi, Shinjuku-ku, Tokyo

160-8582, Japan

Tel +8I 353633829

Fax $+8 I 353790187$

Email hzi05510@nifty.com
Background: Although several functional imaging studies have demonstrated that behavior therapy (BT) modifies the neural circuits involved in the pathogenesis of obsessive-compulsive disorder (OCD), the structural abnormalities underlying BT-resistant OCD remain unknown.

Methods: In this study, we examined the existence of regional structural abnormalities in both the gray matter and the white matter of patients with OCD at baseline using voxel-based morphometry in responders $(n=24)$ and nonresponders $(n=15)$ to subsequent BT. Threedimensional T1-weighted magnetic resonance imaging was performed before the completion of 12 weeks of BT.

Results: Relative to the responders, the nonresponders exhibited significantly smaller gray matter volumes in the right ventromedial prefrontal cortex, the right orbitofrontal cortex, the right precentral gyrus, and the left anterior cingulate cortex. In addition, relative to the responders, the nonresponders exhibited significantly smaller white matter volumes in the left cingulate bundle and the left superior frontal white matter.

Conclusion: These results suggest that the brain structures in several areas, including the orbitofrontal cortex, anterior cingulate cortex, and cingulate bundles, are related to the lack of a response to BT in patients with OCD. The use of a voxel-based morphometry approach may be advantageous to understanding differences in brain abnormalities between responders and nonresponders to BT.

Keywords: OCD, treatment resistance, orbitofrontal cortex

\section{Introduction}

Orbitofrontal-cortex (OFC)-striatal brain circuits are known to be involved in the pathogenesis of obsessive-compulsive disorder (OCD). A large number of functional neuroimaging studies have suggested that several regions, including the basal ganglia, the OFC, and the anterior cingulate cortex (ACC), play major roles in the processing that underlies the emergence of OCD symptoms.

Structural neuroimaging studies in patients with OCD can provide information regarding the structural basis of the pathophysiology of OCD. So far, however, the results of structural neuroimaging studies have been inconsistent. A recent meta-analysis of gray matter (GM) changes based on voxel-based morphometry (VBM), an automated and widely used, whole brain analysis method, has provided evidence of bilateral regional GM volume increases in the lenticular nucleus as well as bilateral regional GM volume decreases in the dorsal medial frontal/anterior cingulate gyri in patients with OCD, compared with healthy controls. ${ }^{1}$ In contrast, another meta-analysis of GM changes based on VBM demonstrated that OCD patients exhibit several areas with smaller volumes 
in the parietofrontal cortical regions, and larger volumes in the putamen and OFC, compared with normal controls. ${ }^{2}$ Yet another meta-analysis based on a region-of-interest method has reported that OCD patients exhibit reduced volumes in the left ACC and right OFC and increased bilateral thalamic volumes, compared with healthy controls. ${ }^{3}$ Finally, a recent mega-analysis in which VBM was used to examine a large number of OCD patients revealed significantly smaller volumes associated with OFC-striatal brain circuits in OCD patients, compared with healthy subjects. ${ }^{4}$ The major reasons for the discrepancies among the above-mentioned studies can probably be attributed to both methodological differences and the heterogeneity of the clinical samples. On the other hand, recent studies have demonstrated that white matter (WM) abnormalities may exist in OCD patients..$^{5-7}$

Several neuroimaging studies have also contributed to an understanding of the neural effects of behavior therapy (BT) in OCD patients. Most of these imaging studies were performed while the patients were in a resting state. Positron emission tomography (PET) studies performed after successful BT have shown a reduction in the right caudate metabolic rate ${ }^{8,9}$ and a reduction in bilateral thalamic activity, while increased activity in the ACC was observed in OCD patients after intensive cognitive BT $(\mathrm{CBT}) .{ }^{10}$ In functional magnetic resonance imaging (MRI) studies, three studies ${ }^{11-13}$ used cognitive provocation paradigms before and after CBT and demonstrated improvements in the function of wide prefrontal-subcortical associations. In terms of identifying predictors of the response to BT, a previous PET study ${ }^{14}$ observed that an elevated pretreatment metabolic activity in the OFC at baseline predicted a better response to BT. Recently, our study ${ }^{15}$ examined the neural effect of BT using single photon emission computed tomography (SPECT) and demonstrated that the baseline regional cerebral blood flow in bilateral OFC was significantly correlated with the change in the Yale-Brown Obsessive-Compulsive Scale (Y-BOCS) score among responders.

However, even after a combination of CBT and pharmacological treatment, half of OCD patients remain treatment resistant. ${ }^{16}$ Thus, the neural mechanisms responsible for treatment resistance should be clarified using not only functional imaging, but also structural imaging. To date, only two studies ${ }^{17,18}$ have used VBM to examine structural changes in OCD patients after CBT, and Lazaro et a ${ }^{17}$ demonstrated a significant increase in the GM volume in all OCD patients (both children and adolescents) after treatment. However, no study has ever examined the structural abnormalities that predict BT resistance.
In this study, we used a VBM approach to examine the existence of regional GM and WM abnormalities at baseline among OCD patients who did and those who did not respond to subsequent BT. We hypothesized that OCD patients who do and those who do not respond to BT may exhibit structural differences in brain regions comprising the cortico-subcortical circuits.

\section{Methods \\ Participants}

Forty-five consecutive patients with OCD who were treated at the Psychiatric Outpatient Department of Nagoya City University Hospital were recruited for this study. All the patients had been diagnosed as having OCD by psychiatrists using the Structured Clinical Interview for DSM-IV (Diagnostic and Statistical Manual of Mental Disorders, Fourth Edition), Patient Version. All the patients had received treatment with serotonin reuptake inhibitors (SRIs) for at least 3 months but had been unresponsive to sufficient doses of SRIs. ${ }^{15,19}$ Therefore, they were judged as being refractory to treatment with SRIs. ${ }^{15,20}$ The SRI doses were maintained during the course of the BT in this study, and the use of psychotropic medication(s) other than SRIs was not allowed. The dose of each SRI was calculated according to a method described elsewhere, ${ }^{21}$ and dose equivalents were calculated using the clomipramine dose $(150 \mathrm{mg} /$ day) as a standard.

The exclusion criteria in this study were as follows: 1) presence of neurological disease, 2) presence of other psychiatric disorders, 3) pregnancy, and 4) previous history of BT. Patients with other axis I disorders were also excluded. Thirty healthy participants with no neurologic or psychiatric disorders who were receiving no medications were recruited as controls.

The purpose and methods of the study were explained in writing to all the participants in an easily understandable manner. This study was conducted with the approval of the Ethics Committee of Nagoya City University Graduate School of Medical Sciences.

\section{Assessment of symptoms}

Patients were assessed using the following evaluation scales before and at the end of treatment. The general severity of OCD was assessed by psychiatrists (who were blinded to the treatment group) using the Y-BOCS. ${ }^{22,23}$ Patients with a reduction in their global Y-BOCS score of $40 \%$ or greater were judged as being responsive to BT, as defined in previous papers. ${ }^{15,24}$ OCD subtypes were identified using the 
Y-BOCS checklist. ${ }^{25}$ Depression and anxiety were assessed using two self-reported questionnaires: the Beck Depression Inventory-II (BDI-II) ${ }^{26}$ and the State-Trait Anxiety Inventory (STAI), respectively. ${ }^{27}$

\section{Treatment}

The treatment program was applied not to groups, but to each patient individually, based on the treatment strategy outlined in a manual. ${ }^{28}$ Weekly sessions were conducted 12 times. The psychiatrists who provided the treatments had at least 5 years of clinical experience using the exposure and response prevention technique. The treatments included the following three processes: 1) psycho-education regarding the disease and its treatment, 2) treatment planning by a therapist through an analysis of the behavior and anxiety experienced during each patient's daily life, and 3) the initiation of exposure and response prevention technique based on the treatment plan. Exposure exercises were organized hierarchically according to the level of anxiety provoked by situations to which the patient was exposed. Exposure exercises were administered using situations provoking mild to severe anxiety, in that order. After exposure to anxietyprovoking situations, the patients received response prevention until the level of anxiety provoked by the exposure decreased sufficiently. Weekly homework assignments were given to each patient.

\section{MRI image acquisition}

At the baseline of treatment, MRI images were obtained using a 1.5-T MRI system (Gyoro Scan Interu; Philips Medical Systems, Best, the Netherlands). The scanning parameters for the three-dimensional-T1-weighted turbo filled echo sequences were acquired using the following parameters: echo time $=3.90 \mathrm{~ms}$; repetition time $=8.4 \mathrm{~ms}$; inversion time $=1,000 \mathrm{~ms}$; flip angle $=7^{\circ}$; acquisition matrix $=256 \times$ 256; field of view $=256 \mathrm{~mm}$, section thickness $=1 \mathrm{~mm}$; and voxel size $=1.0 \mathrm{~mm} \times 1.0 \mathrm{~mm} \times 1.0 \mathrm{~mm}$. All the MRI scans were acquired at the Department of Radiology, Nagoya City University Hospital.

\section{VBM protocol}

VBM was performed using the VBM 8-toolbox (http://dbm. neuro.uni-jena.de/vbm) implemented in SPM8 (http://www. fil.ion.ucl.ac.uk/spm) running on Matlab 7.5 (Mathworks Inv., Sherborn, MA, USA) using the default parameters. Each 3D-MRI T1-weighted image was segmented into GM, WM, and cerebrospinal fluid. Then, the segmented images were spatially normalized to the same stereotaxic space using
DARTEL (diffeomorphic anatomical registration through an exponentiated Lie algebra) algorithm. ${ }^{29}$ The voxel values were modulated using Jacobian determinants for the nonlinear components, and were smoothed using a Gaussian kernel of $8 \mathrm{~mm}$ full width at half maximum.

\section{Statistical analysis}

We examined differences in the demographic and clinical variables among the three groups using an analysis of variance. The statistical changes in the treatment effects of BT among OCD subjects were analyzed using paired $t$-tests for both the responders and the nonresponders.

A one-way analysis of variance analysis was then used to investigate the regional differences in the GM or WM volumes among three groups (responders, nonresponders, and healthy participants) using VPM 8. If the group effects were significant, separate post hoc analyses of every two groups (responders vs nonresponders, nonresponders vs healthy participants, and responders vs healthy participants) were performed using VPM 8 to detect regional differences in the GM or WM volumes.

Finally, we performed a multiple regression analysis to test the relationships between either the regional GM or WM volumes at baseline and the change in the Y-BOCS score among not only the responders, but also among the nonresponders. For all these analyses, patient age was used as a nuisance covariate. In this analysis, we estimated the statistical significance level as a false discovery rate ${ }^{30}$ was set at 0.05 to correct for multiple comparisons. We set the cluster extent threshold as 100 voxels to reduce possible noise. We also converted the cluster locations from coordinates related to the Montreal Neurologic Institute atlas system to coordinates associated with the Talairach atlas.

\section{Results Clinical characteristics of the participants} Six of the 45 patients who initially provided consent were withdrawn from the study because they were transferred to another clinic (two patients) or were unwilling to undergo an MRI examination (four patients). All the participants were right-handed. Twenty-four of the 39 patients responded to BT, while the remaining $15 \mathrm{did}$ not. The clinical characteristics of the participants are shown in Table 1. No significant differences in the clinical characteristics, including the age, sex ratio, and medical history, were observed between the responders and the nonresponders. Furthermore, no significant differences in the degree of compulsion, depression, and/or anxiety were observed between the two groups. The demographic 
Table I Demographic and clinical findings of OCD patients $(n=39)$ and healthy participants $(n=30)$

\begin{tabular}{|c|c|c|c|c|}
\hline & $\begin{array}{l}\text { Responders }(n=24) \\
\text { (A) }\end{array}$ & $\begin{array}{l}\text { Nonresponders }(n=15) \\
\text { (B) }\end{array}$ & $\begin{array}{l}\text { Healthy participants }(n=30) \\
\text { (C) }\end{array}$ & $P$ \\
\hline & Mean (SD) & Mean (SD) & Mean (SD) & \\
\hline Age, years & $35.7(7.2)$ & $32.5(7.7)$ & $32.5(6.7)$ & 0.208 \\
\hline Sex (male, \%) & 45.8 & 46.6 & 46.6 & ns \\
\hline Years of education & $14.4(1.5)$ & I3.6 (I.8) & I4.I (I.5) & 0.322 \\
\hline Duration of illness, years & $6.3(3.2)$ & $5.2(2.5)$ & $(-)$ & 0.250 \\
\hline SRI, mg/day (clomipramine equivalent) & $220.8(25.1)$ & $243.3(41.6)$ & $(-)$ & 0.070 \\
\hline BDI-II & $16.1(6.3)$ & $15.4(7.3)$ & $1.9(1.2)$ & $<0.001 \mathrm{~A}=\mathrm{B}>\mathrm{C}$ \\
\hline \multicolumn{5}{|l|}{ STAI } \\
\hline State anxiety & $49.6(10.8)$ & $55.8(I I .2)$ & $27.1(4.5)$ & $<0.001 \mathrm{~A}=\mathrm{B}>\mathrm{C}$ \\
\hline Trait anxiety & $54.2(12.1)$ & $61.1(11.8)$ & $33.6(4.1)$ & $<0.001 \mathrm{~A}=\mathrm{B}>\mathrm{C}$ \\
\hline \multicolumn{5}{|l|}{ Y-BOCS } \\
\hline Total & $33.3(4.2)$ & $33.1(5.3)$ & $(-)$ & 0.844 \\
\hline Obsessions & $16.7(2.1)$ & $16.8(2.9)$ & $(-)$ & 0.927 \\
\hline Compulsions & $16.5(2.6)$ & $16.2(2.7)$ & $(-)$ & 0.644 \\
\hline \multicolumn{5}{|l|}{ Brain volumes } \\
\hline TIV, mL & I,604.5 (87.9) & I,568.3 (I24.I) & I,6II.4 (I34.6) & 0.501 \\
\hline GM volume, $\mathrm{mL}$ & $669.8(10.5)$ & $642.0(13.3)$ & $655.4(9.4)$ & 0.256 \\
\hline $\mathrm{WM}$ volume, $\mathrm{mL}$ & $456.6(47.2)$ & $496.5(85.3)$ & $469.5(58.1)$ & 0.158 \\
\hline
\end{tabular}

Notes: The male/female ratio was compared using a chi-square test $\left(\chi^{2}\right)$. (-) No data.

Abbreviations: BDI-II, Beck Depression Inventory-II; GM, gray matter; ns, not significant; OCD, obsessive-compulsive disorder; SD, standard deviation; SRI, serotonin reuptake inhibitors; STAI, State-Trait Anxiety Inventory; TIV, total intracranial volume; WM, white matter; Y-BOCS, Yale-Brown Obsessive Compulsive Scale.

characteristics of the healthy participants were similar to those of the patients with OCD. No significant differences were observed in the total intracranial volume, global GM volume, or global WM volume among the three groups.

Based on the Y-BOCS symptom checklist, ${ }^{25}$ the following OCD symptom types were identified: among the responders with OCD, contamination/cleaning was the major symptom in 16 patients, aggressive/checking was the major symptom in five patients, and symmetry/ordering was the major symptom in three patients. Among the nonresponders with OCD, contamination/cleaning was the major symptom in eight patients, aggressive/checking was the major symptom in four patients, and symmetry/ordering was the major symptom in three patients. No significant differences in any symptom dimension were observed between the two OCD groups (contamination/cleaning $t_{37}=1.135, P=0.183$; aggressive/ checking $t_{37}=1.415, P=0.165$; symmetry/ordering $t_{37}=1.896$, $P=0.067)$. Sexual/religious symptoms or hoarding symptoms were not identified in either OCD group.

\section{Comparison of the clinical findings before and after treatment}

Differences in the results of the clinical assessments performed before and after BT are shown in Table 2. The Y-BOCS scores changed significantly after treatment among both the OCD patients who responded to BT and those who did not respond to BT. However, while the responders had a mean Y-BOCS score reduction of $52.2 \% \pm 7.4 \%$, the nonresponders had a mean $\mathrm{Y}$-BOCS score reduction of as low as $10.5 \% \pm 8.4 \%$. No significant changes in the mean BDI-II scores after treatment were observed for either the responders or the nonresponders.

Table 2 Clinical findings before and after behavior therapy in the OCD groups $(n=39)$

\begin{tabular}{|c|c|c|c|}
\hline & Pre-treatment & Post-treatment & $P$ \\
\hline & Mean (SD) & Mean (SD) & \\
\hline \multicolumn{4}{|c|}{ Responders ( $n=24)$} \\
\hline \multicolumn{4}{|l|}{ Y-BOCS } \\
\hline Total & $33.3(4.2)$ & $16.0(3.3)$ & $<0.001$ \\
\hline Obsessions & $16.7(2.1)$ & $8.1(1.7)$ & $<0.001$ \\
\hline Compulsions & $16.5(2.6)$ & $7.8(2.0)$ & $<0.001$ \\
\hline BDI-II & I6.I (6.3) & $15.9(5.7)$ & 0.698 \\
\hline \multicolumn{4}{|l|}{ STAI } \\
\hline State anxiety & $51.5(12.2)$ & $38.9(9.5)$ & $<0.001$ \\
\hline Trait anxiety & $52.2(11.0)$ & $45.5(8.9)$ & $<0.001$ \\
\hline \multicolumn{4}{|c|}{ Nonresponders $(n=15)$} \\
\hline \multicolumn{4}{|l|}{ Y-BOCS } \\
\hline Total & $33.0(5.3)$ & $30.0(5.5)$ & 0.012 \\
\hline Obsessions & $16.8(2.9)$ & $15.6(3.4)$ & 0.031 \\
\hline Compulsions & $16.2(2.7)$ & $14.9(2.3)$ & 0.029 \\
\hline BDI-II & $15.4(7.3)$ & $15.0(6.8)$ & 0.417 \\
\hline \multicolumn{4}{|l|}{ STAI } \\
\hline State anxiety & $55.8(1 \mathrm{I} .2)$ & $54.8(1 \mathrm{I} .2)$ & 0.610 \\
\hline Trait anxiety & $61.1(11.8)$ & $60.4(12.6)$ & 0.056 \\
\hline
\end{tabular}

Abbreviations: BDI-II, Beck Depression Inventory-II; OCD, obsessive-compulsive disorder; SD, standard deviation; STAl, State-Trait Anxiety Inventory; Y-BOCS, YaleBrown Obsessive Compulsive Scale. 
Table 3 Areas with smaller regional GM and WM volumes in nonresponder OCD patients $(n=15)$ than in responder OCD patients $(n=24)$

\begin{tabular}{|c|c|c|c|c|c|c|}
\hline \multirow[t]{2}{*}{ Region } & \multirow[t]{2}{*}{ Brodmann's area } & \multicolumn{3}{|c|}{ Talairach coordinate $(\mathrm{mm})$} & \multirow[t]{2}{*}{ Voxels in cluster } & \multirow[t]{2}{*}{$T$-value } \\
\hline & & $\mathbf{x}$ & $y$ & $\mathbf{z}$ & & \\
\hline Rt ventromedial prefrontal cortex & 25 & 1 & 14 & -14 & 3,476 & 6.55 \\
\hline Rt orbitofrontal cortex & 11 & 8 & 25 & -16 & 3,476 & 4.93 \\
\hline Rt orbitofrontal cortex & 11 & 7 & 25 & -24 & 3,476 & 3.85 \\
\hline Rt precentral gyrus & 6 & 56 & 2 & 39 & 944 & 6.39 \\
\hline Rt precentral gyrus & 6 & 60 & -3 & 28 & 944 & 4.22 \\
\hline Rt precentral gyrus & 6 & 58 & -5 & 37 & 944 & 3.69 \\
\hline Lt anterior cingulate cortex & 24 & -3 & 3 & 27 & 805 & 5.06 \\
\hline Lt cingulate bundle & & -4 & 4 & 38 & $\mathrm{I}, 328$ & 4.99 \\
\hline Lt cingulate bundle & & -1 & 18 & 26 & 1,328 & 4.85 \\
\hline Lt cingulate bundle & & -1 & 11 & 30 & 1,328 & 4.50 \\
\hline Lt superior frontal white matter & & -31 & -13 & 48 & 928 & 5.82 \\
\hline Lt superior frontal white matter & & -25 & -5 & 47 & 928 & 4.11 \\
\hline
\end{tabular}

Notes: FDR set at 0.05 . Results of a voxel-wise SPM analysis.

Abbreviations: FDR, false discovery rate; GM, gray matter; Lt, left; OCD, obsessive-compulsive disorder; Rt, right; SPM, statistical parametric mapping; WM, white matter.

\section{Results of VBM analysis}

Among the three groups, the main effects on the GM volumes were significant for the right ventromedial prefrontal cortex, the right $\mathrm{OFC}$, the right precentral gyrus, the left ACC, the right thalamus, the right caudate, the left posterior cingulate cortex, and the left dorsolateral prefrontal cortex (DLPFC). In addition, the main effects on the WM volumes were significant for the left cingulate bundle, the left superior WM, the right inferior frontal WM, the left cingulate bundle, and the left superior frontal WM.

A post hoc two-sample $t$-test (responders vs nonresponders) (Table 3 and Figure 1) showed both GM and
WM volume differences between the responders and the nonresponders. At the time of the baseline assessments, the nonresponders exhibited significantly smaller GM volumes in the right ventromedial prefrontal cortex (Brodmann 25), the right OFC (Brodmann 11), the right precentral gyrus (Brodmann 6), and the left ACC (Brodmann 24), compared with the responders. No significantly larger GM volumes were identified for any brain region among the nonresponders, compared with the responders. The nonresponders exhibited significantly smaller WM volumes in the left cingulate bundle and the left superior WM, compared with the responders. No significantly larger WM volumes were identified for
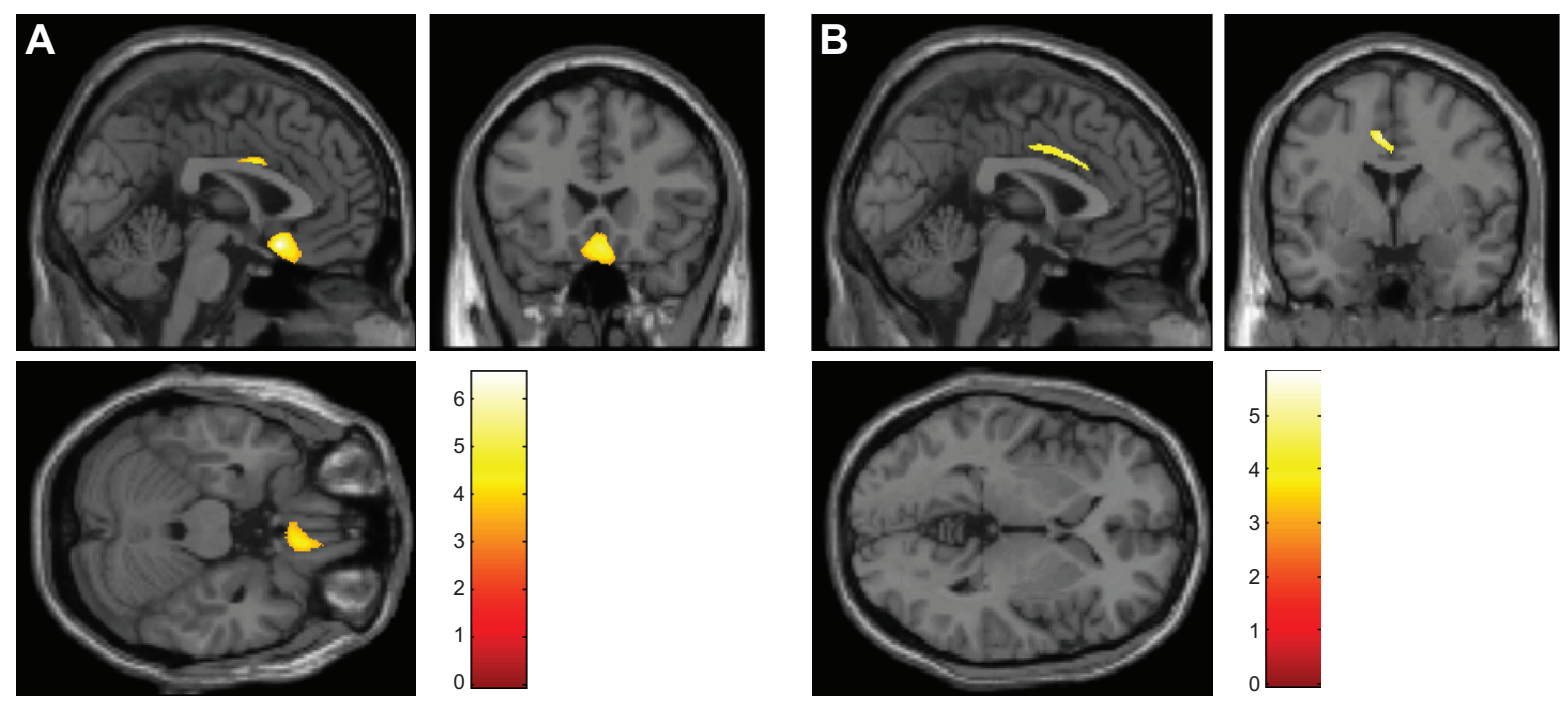

Figure I (A) Smaller regional GM volumes were observed for the right orbitofrontal cortex and left cingulate cortex in nonresponder OCD patients ( $\mathrm{n}=\mathrm{I5}$ ), compared with responder OCD patients $(n=24)$. (B) Smaller regional WM volumes were observed for the left cingulate bundle in nonresponder OCD patients ( $n=15)$, compared with responder OCD patients $(n=24)$.

Abbreviations: GM, gray matter; OCD, obsessive-compulsive disorder; WM, white matter. 
any brain region among the nonresponders, compared with the responders. In addition, no significant correlations were observed between the regional GM or WM volumes at baseline and the reduction in the Y-BOCS score among either the nonresponders or the responders. Furthermore, we performed a regression analysis to identify either regional GM or WM volumes that might be good predictors of clinical symptoms in OCD patients. In this regression analysis, GM density data for several areas (the right ventromedial prefrontal cortex, the right OFC, the right precentral gyrus, and the left ACC) were used as independent variables, and several clinical features (the reduction change in the Y-BOCS score, the reduction change in the BDI-II score, and the reduction change in the STAI) were used as dependent variables for separate analyses of both the nonresponders and the responders. In addition, WM density data for two areas (the left cingulate bundle and the left superior WM) were used as independent variables, and several clinical features (the reduction change in the Y-BOCS score, the reduction change in the BDI-II score, and the reduction change in the STAI) were used as dependent variables for separate analyses of both the nonresponders and the responders. However, no significant relationships between the GM/WM density data and the clinical features were observed among the nonresponders or the responders.

A post hoc two-sample $t$-test (nonresponders vs healthy participants) (Table 4) showed both GM and WM volume differences between the nonresponders with OCD and the healthy participants. At the time of the baseline assessments, the nonresponders exhibited significantly smaller GM volumes in several brain areas including the right thalamus, the right caudate, and the left posterior cingulate cortex, compared with the healthy participants. No significantly larger GM volumes were identified for any brain region among the nonresponders, compared with the healthy participants. Also, the nonresponders exhibited significantly smaller WM volumes in the right inferior frontal WM and the left cingulate bundle at the time of the baseline assessments, compared with the healthy participants. No significantly larger WM volumes were observed for any brain region among the nonresponders, compared with the healthy participants.

A post hoc two-sample $t$-test (responders vs healthy participants) (Table 5) showed both GM and WM volume differences between the responders with OCD and healthy participants. At the time of the baseline assessments, the responders exhibited significantly smaller GM volumes in several brain areas including the left DLPFC (Brodmann 8), compared with the healthy participants. No significantly larger GM volumes were observed for any brain region among the responders, compared with the healthy participants. In addition, the responders with OCD exhibited significantly smaller WM volumes in the left superior frontal WM at the time of the baseline assessments, compared with the healthy participants. No significantly larger WM volumes were observed for any brain region among the responders, compared with the healthy participants.

\section{Discussion}

A unique MRI study, in which OCD patients were examined using VBM, was performed to determine whether any baseline structural differences exist between responders and nonresponders to BT. Compared with responders, nonresponders exhibited smaller GM volumes in several clinically important areas, such as the right ventromedial prefrontal cortex (Brodmann 25), the right OFC (Brodmann 11), and the left ACC (Brodmann 24), all of which have been implicated in the pathogenesis of OCD. In addition, nonresponders exhibited smaller WM volumes in the left cingulate bundle and the left superior frontal WM, two key regions that have also been implicated in WM abnormalities in patients with OCD.

Table 4 Areas with smaller regional GM and WM volumes in nonresponder OCD patients $(n=15)$ than in healthy participants $(n=30)$

\begin{tabular}{|c|c|c|c|c|c|c|}
\hline \multirow[t]{2}{*}{ Region } & \multirow[t]{2}{*}{ Brodmann's area } & \multicolumn{3}{|c|}{ Talairach coordinate $(\mathrm{mm})$} & \multirow[t]{2}{*}{ Voxels in cluster } & \multirow[t]{2}{*}{$T$-value } \\
\hline & & $\mathbf{x}$ & y & $\mathbf{z}$ & & \\
\hline Rt thalamus & & 3 & -5 & 0 & 2,486 & 4.87 \\
\hline Rt caudate & & 6 & 0 & 4 & 2,486 & 4.54 \\
\hline Lt posterior cingulate cortex & 30 & -21 & -59 & 9 & $\mathrm{I}, 707$ & 8.28 \\
\hline Lt posterior cingulate cortex & 30 & -14 & -65 & 10 & ।,707 & 4.79 \\
\hline Rt inferior frontal white matter & & 17 & 57 & -13 & $\mathrm{I}, 178$ & 5.90 \\
\hline Rt inferior frontal white matter & & 11 & 53 & -13 & I, I 78 & 5.14 \\
\hline Rt inferior frontal white matter & & 13 & 40 & -19 & $\mathrm{I}, 178$ & 4.84 \\
\hline Lt cingulate bundle & & -25 & -60 & 14 & $\mathrm{I}, \mathrm{I} 42$ & 4.51 \\
\hline
\end{tabular}

Notes: FDR set at 0.05. Results of a voxel-wise SPM analysis.

Abbreviations: FDR, false discovery rate; GM, gray matter; Lt, left; OCD, obsessive-compulsive disorder; Rt, right; SPM, statistical parametric mapping; WM, white matter. 
Table 5 Areas with smaller regional GM and WM volumes in responder OCD patients $(n=24)$ than in healthy participants ( $n=30)$

\begin{tabular}{lllllll}
\hline Region & Brodmann's area & \multicolumn{2}{c}{ Talairach coordinate $\mathbf{( m m )}$} & & \multirow{2}{*}{ Voxels in cluster } \\
\cline { 3 - 5 } & & $\mathbf{x}$ & $\mathbf{y}$ & $\mathbf{z}$ & & \\
\hline Lt dorsolateral prefrontal & 8 & -52 & 10 & 40 & 848 & 7.17 \\
Lt dorsolateral prefrontal & 8 & -39 & 21 & 42 & 848 & 6.50 \\
Lt superior frontal white matter & & -30 & -11 & 47 & 948 & 6.37 \\
Lt superior frontal white matter & & -36 & -17 & 55 & 948 & \\
\hline
\end{tabular}

Notes: FDR set at 0.05 . Results of a voxel-wise SPM analysis.

Abbreviations: FDR, false discovery rate; GM, gray matter; Lt, left; OCD, obsessive-compulsive disorder; SPM, statistical parametric mapping; WM, white matter.

The most important finding was that compared with the responders, the nonresponders had smaller GM volumes in the right ventromedial prefrontal cortex and right OFC. Previous VBM studies have shown volume alterations in either the OFC or the medial frontal cortex; although some studies have reported smaller GM volumes in the OFC, ${ }^{11-34}$ other studies ${ }^{35,36}$ have reported larger OFC volumes. Interestingly, a recent volumetric MRI study (using a regionof-interest approach) in patients with OCD demonstrated that smaller OFC volumes were associated with treatment resistance to selective SRIs. ${ }^{37}$ The OFC is thought to play important roles in decision-making ability, reversal learning, and fear extinction..$^{38,39}$ The core features of OCD patients with ineffective behavior adaptations to day-to-day activities are characterized by such cognitive dysfunctions. Although BT involves various cognitive aspects, which aspects of cognitive dysfunction can be improved by BT in patients with OCD symptoms remains unclear. However, a recent functional MRI study using a reversal learning task demonstrated an influence on brain activity after CBT for OCD. ${ }^{13}$ In addition, several neuroimaging studies (PET and SPECT) have suggested that OFC function may be associated with the response to BT in OCD patients. ${ }^{14,15}$ Recently, Huyser et $\mathrm{al}^{40,41}$ demonstrated that the GM volume in the OFC increased after $\mathrm{CBT}$ in pediatric patients with $\mathrm{OCD}$, and the increased GM volume changes in the OFC persisted throughout a 2-year follow-up period. They suggested that an increased OFC volume might arise as a result of a compensatory mechanism triggered by CBT. Unfortunately, we could not perform a second MRI assessment after the completion of BT. Thus, whether a correlation exists between the volume changes in the right ventromedial prefrontal cortex and the right $\mathrm{OFC}$ and an improvement in the symptoms of OCD patients remains unclear. However, both the OFC and the adjacent ventromedial prefrontal cortex may be involved in fear extinction, which is thought to be at the core of BT. ${ }^{18,38,39}$ Thus, relative to responders to $\mathrm{BT}$, the smaller volume in the OFC observed in nonresponders might be a marker of vulnerability underlying the biological mechanisms responsible for the lack of a response to BT. A BT-specific function might be associated with the role of the OFC..$^{15}$

Previous studies ${ }^{14,15}$ have suggested that an elevated pretreatment activity in the OFC at baseline predicted a better response to BT. However, in the current study, we did not observe a significant correlation between the change in the Y-BOCS score and the regional GM or WM volumes at baseline among either the responders or the nonresponders. Using VBM, Hoexter et a ${ }^{18}$ demonstrated that in CBT-treated but medication-free OCD patients, a larger pretreatment GM volume in the medial prefrontal cortex was significantly correlated with a reduction in the Y-BOCS score. In addition, in a fluoxetine-treatment group, a smaller GM volume in the OFC was significantly correlated with a reduction in the Y-BOCS score. However, when both types of treatments were analyzed together, no significant correlations were found. Unlike the study by Hoexter et al, ${ }^{18}$ in our study, all the OCD patients were taking SRIs at the study baseline, and the patients continued to use SRIs throughout the BT treatment, although the SRI doses were not altered during the course of the BT. In addition, the severity of OCD was higher in our study than in the study by Hoexter et $\mathrm{al}^{18}$ (Y-BOCS total score of 33 in our study vs Y-BOCS total score of 25 in the study by Hoexter et $\mathrm{al}^{18}$ ). Therefore, unlike the results of previous functional neuroimaging studies (including PET, SPECT, and functional MRI), the treatment responses of the OCD patients in our study may be difficult to predict in terms of structural abnormalities visualized using MRI. Further studies examining drug-naïve patients with $\mathrm{OCD}$ who exhibit a mild level of disease severity are needed to clarify the prediction of BT response using VBM.

Consistent with most previous VBM studies, our study showed a smaller GM volume in the left ACC of nonresponders, compared with that of responders. In addition, similar to the results of several previous studies, ${ }^{31,42}$ we observed a smaller GM volume in the right precentral gyrus (Brodmann 25). The ACC is known to be involved in attention control. 
Some evidence suggests that the ACC in OCD patients is associated with action monitoring in processing competing information, ${ }^{11}$ error detection, ${ }^{11}$ and the expression of fear responses. ${ }^{38}$ The precentral areas are assumed to be related to the coordination of complex motor activities. ${ }^{42}$ Thus, structural abnormalities in both the left ACC and the right precentral areas may result in dysfunctions in the cognitive abilities required for a response to $\mathrm{BT}$ among OCD patients.

Regarding WM abnormalities, the nonresponders had smaller WM volumes in the left cingulate bundle, compared with the responders. Most previous studies ${ }^{5-7,43,44}$ examining WM pathology in patients with OCD have used diffusion tensor imaging (DTI) and have revealed microstructural WM abnormalities in the cingulate bundles. An anterior cingulotomy is known to be an effective neurosurgical treatment for treatment-refractory OCD, disrupting the connections between the frontal lobes and the subcortical structures. ${ }^{45}$ Thus, our study supports the hypothesis that the cingulate bundle is one of the main WM tracts involved in the pathogenesis of OCD. Furthermore, our study showed a smaller WM volume in the left cingulate bundle in the nonresponders, compared with the healthy participants. The reason for this finding is unclear. Additional studies using DTI are needed to clarify the differences in WM abnormalities among OCD patients, including both responders and nonresponders, and normal controls.

Although significant structural differences in either the thalamus or the caudate were not observed between the responders and the nonresponders, the nonresponders exhibited significantly smaller GM volumes on the right side of both areas, compared with the healthy participants. Both the thalamus and the caudate are key structures in cortico-basal ganglia-thalamic-cortical loops, which are known neuroanatomical modes of OCD. More importantly, previous neuroimaging studies have suggested the modulation of neural function in these two regions in response to $\mathrm{BT} .{ }^{8-10}$ Thus, the GM volume reductions in these two areas in nonresponders may reflect the dysfunction of abilities required for a response to BT among patients with OCD. A smaller GM volume in bilateral DLPFC was identified only among the responders, compared with the healthy participants. Recently, the DLPFC has been considered to have an especially important role in executive functions associated with not only planning, but also working memory in patients with OCD. ${ }^{46}$ The DLPFC is thought to be involved in the dorsolateral prefrontal-striatal loop, one of two independent frontal-striatal networks. However, only a few studies using VBM have detected volume abnormalities in the DLPFC. ${ }^{33,47}$ In addition, with the exception of one study, ${ }^{11}$ previous neuroimaging studies examining the effects of BT in patients with OCD patients failed to detect regional changes in the DLPFC. These findings suggest that the DLPFC may contribute to the biological mechanisms responsible for the lack of a response to BT.

Regarding laterality, we observed that while a smaller GM volume was mainly observed on the left side in both BT responders and nonresponders, compared with that in healthy participants, some right-sided frontal GM abnormalities were observed in the BT nonresponders, compared with the responders. The most consistent finding among previous functional neuroimaging studies examining $\mathrm{CBT}^{8-11,13,15}$ is that the predominantly right-sided neural activity within the frontostriatal circuit improved in response to CBT. Using structural imaging studies of adolescents with OCD, Hoexter et $\mathrm{al}^{18}$ demonstrated that the right side of the medial prefrontal cortex may be a key region in response to $\mathrm{CBT}$. Also, Schwartz et $\mathrm{al}^{9}$ speculated that the function of the right side of the cortex might be more severely damaged than that on the left side. Taken together, these results suggest that a right laterality in the frontal cortex, rather than a left laterality, may be associated with a lack of response to BT. However, regarding the WM area, we observed a smaller left-side WM volume in the BT nonresponders, compared with the responders. Thus, if we include abnormalities in not only the GM area, but also the WM area, any speculation regarding a laterality effect in response to BT must be interpreted with caution. As far as we know, the neural effect of the BT response has not yet been examined using DTI.

Finally, we must address several limitations. First, the patients with OCD in this study were not drug naïve and were taking SRIs at the study baseline prior to MRI scanning. As suggested by several studies, ${ }^{48,49}$ serotonergic pharmacotherapy may affect regional brain volumes. In our study, however, the average daily dose of SRIs was not significantly different between the responders and the nonresponders with OCD. In addition, before the BT, all the OCD patients were judged as being refractory to treatment with SRIs. The SRI doses were also maintained during the course of the BT. Thus, SRI therapy was unlikely to have had a major effect on the treatment effect of the BT. Second, although we performed the clinical assessments (eg, Y-BOCS and BDI-II) before and after treatment, we could not perform a post-treatment MRI study after the last BT treatment. Thus, we could not examine the correlation between the GM/WM volume changes and the improvement of symptoms after BT in the OCD patients. In addition, all the participants 
underwent MRI using a 1.5-T MRI system, rather than a 3.0-T system. Thus, whether structural brain changes may occur in patients with OCD after BT remains unclear. Further longitudinal studies are needed to clarify the effects of successful BT on both the GM and WM volumes in patients with OCD. Third, the duration of the BT program (weekly sessions, 12 weeks in total) in our study was shorter than that reported in other studies (Lazaro et a ${ }^{17}$ weekly sessions for 6 months; Huyser et $\mathrm{al}^{40} 16$ weekly sessions). A modified and improved treatment program may be required in future studies to clarify the neural effects of BT in OCD patients. Fourth, we did not include a placebo control group. Therefore, we had to rely on single-arm within-group changes to examine the effects of BT. Fifth, a recent structural MRI study has shown that several major symptom dimensions are associated with specific brain abnormalities. ${ }^{34}$ In addition, specific symptom dimensions are related to a poor outcome of BT. ${ }^{50}$ However, no significant differences in symptom dimensions were observed between the responders and the nonresponders with OCD. Additional large-scale studies examining OCD patients are needed to clarify the effect of different types of symptom dimensions on treatment.

Despite these limitations, the results of the current study have provided clinically important findings: among the patients with OCD, nonresponders to BT have smaller baseline volumes of not only the GM in the right ventromedial prefrontal cortex, right OFC, and left ACC, but also the WM in the left cingulate bundle and left external capsule. Our study implicates these areas in the pathogenesis of OCD as it relates to the role of BT. The findings of this study are likely to contribute to a better understanding of brain abnormalities as biological markers of vulnerability in terms of the likelihood of a response to BT among patients with OCD.

\section{Acknowledgments}

The authors gratefully acknowledge a Grant-in-Aid for Scientific Research (c) (24530884) from the Ministry of Education, Culture, Sports, Sciences and Technology in Japan.

\section{Disclosure}

The authors report no conflicts of interest in this work.

\section{References}

1. Radua J, Mataix-Cols D. Voxel-wise meta-analysis of grey matter changes in obsessive-compulsive disorder. Br J Psychiatry. 2009;195(5): 393-402.

2. Rotge JY, Langbour N, Guehl D, et al. Gray matter alterations in obsessive-compulsive disorder: an anatomic likelihood estimation metaanalysis. Neuropsychopharmacology. 2010;35(3):686-691.
3. Rotge JY, Langbour N, Jaafari N, et al. Anatomical alterations and symptom-related functional activity in obsessive-compulsive disorder are correlated in the lateral orbitofrontal cortex. Biol Psychiatry. 2010;67(7):e37-e38.

4. de Wit SJ, Alonso P, Schweren L, et al. Multicenter voxel-based morphometry mega-analysis of structural brain scans in obsessivecompulsive disorder. Am J Psychiatry. 2014;171(3):340-349.

5. Szeszko PR, Ardekani BA, Ashtari M, et al. White matter abnormalities in obsessive-compulsive disorder: a diffusion tensor imaging study. Arch Gen Psychiatry. 2005;62(7):782-790.

6. Nakamae T, Narumoto J, Sakai Y, et al. Diffusion tensor imaging and tract-based spatial statistics in obsessive-compulsive disorder. J Psychiatr Res. 2011;45(5):687-690.

7. Garibotto V, Scifo P, Gorini A, et al. Disorganization of anatomical connectivity in obsessive compulsive disorder: a multi-parameter diffusion tensor imaging study in a subpopulation of patients. Neurobiol Dis. 2010;37(2):468-476.

8. Baxter LR Jr, Schwartz JM, Bergman KS, et al. Caudate glucose metabolic rate changes with both drug and behavior therapy for obsessive-compulsive disorder. Arch Gen Psychiatry. 1992;49(9): 681-689.

9. Schwartz JM, Stoessel PW, Baxter LR Jr, Martin KM, Phelps ME. Systematic changes in cerebral glucose metabolic rate after successful behavior modification treatment of obsessive-compulsive disorder. Arch Gen Psychiatry. 1996;53(2):109-113.

10. Saxena S, Gorbis E, O'Neill J, et al. Rapid effects of brief intensive cognitive-behavioral therapy on brain glucose metabolism in obsessivecompulsive disorder. Mol Psychiatry. 2009;14(2):197-205.

11. Nakao T, Nakagawa A, Yoshiura T, et al. Brain activation of patients with obsessive-compulsive disorder during neuropsychological and symptom provocation tasks before and after symptom improvement: a functional magnetic resonance imaging study. Biol Psychiatry. 2005;57(8):901-910.

12. Nabeyama M, Nakagawa A, Yoshiura T, et al. Functional MRI study of brain activation alterations in patients with obsessivecompulsive disorder after symptom improvement. Psychiatry Res. 2008;163(3):236-247.

13. Freyer T, Kloppel S, Tuscher O, et al. Frontostriatal activation in patients with obsessive-compulsive disorder before and after cognitive behavioral therapy. Psychol Med. 2011;41(1):207-216.

14. Brody AL, Saxena S, Schwartz JM, et al. FDG-PET predictors of response to behavioral therapy and pharmacotherapy in obsessive compulsive disorder. Psychiatry Res. 1998;84(1):1-6.

15. Yamanishi T, Nakaaki S, Omori IM, et al. Changes after behavior therapy among responsive and nonresponsive patients with obsessivecompulsive disorder. Psychiatry Res. 2009;172(3):242-250.

16. Denys D, Mantione M, Figee M, et al. Deep brain stimulation of the nucleus accumbens for treatment-refractory obsessive-compulsive disorder. Arch Gen Psychiatry. 2010;67(10):1061-1068.

17. Lazaro L, Bargallo N, Castro-Fornieles J, et al. Brain changes in children and adolescents with obsessive-compulsive disorder before and after treatment: a voxel-based morphometric MRI study. Psychiatry Res. 2009;172(2):140-146.

18. Hoexter MQ, Dougherty DD, Shavitt RG, et al. Differential prefrontal gray matter correlates of treatment response to fluoxetine or cognitivebehavioral therapy in obsessive-compulsive disorder. Eur Neuropsychopharmacol. 2013;23(7):569-580.

19. Tolin DF, Maltby N, Diefenbach GJ, Hannan SE, Worhunsky P. Cognitive-behavioral therapy for medication nonresponders with obsessive-compulsive disorder: a wait-list-controlled open trial. J Clin Psychiatry. 2004;65(7):922-931.

20. Pallanti S, Hollander E, Goodman WK. A qualitative analysis of nonresponse: management of treatment-refractory obsessive-compulsive disorder. J Clin Psychiatry. 2004;65 Suppl 14:6-10.

21. Bollini P, Pampallona S, Tibaldi G, Kupelnick B, Munizza C. Effectiveness of antidepressants. Meta-analysis of dose-effect relationships in randomised clinical trials. Br J Psychiatry. 1999;174:297-303. 
22. Goodman WK, Price LH, Rasmussen SA, et al. The Yale-Brown Obsessive Compulsive Scale. I. Development, use, and reliability. Arch Gen Psychiatry. 1989;46:1006-1011.

23. Goodman WK, Price LH, Rasmussen SA, et al. The Yale-Brown Obsessive Compulsive Scale. II. Validity. Arch Gen Psychiatry. 1989; 46:1012-1016.

24. Tolin DF, Abramowitz JS, Diefenbach GJ. Defining response in clinical trials for obsessive-compulsive disorder: a signal detection analysis of the Yale-Brown obsessive compulsive scale. J Clin Psychiatry. 2005;66(12):1549-1557.

25. Mataix-Cols D, Rauch SL, Manzo PA, Jenike MA, Baer L. Use of factor-analyzed symptom dimensions to predict outcome with serotonin reuptake inhibitors and placebo in the treatment of obsessivecompulsive disorder. Am J Psychiatry. 1999;156(9):1409-1416.

26. Beck AT, Steer RA, Ball R, Ranieri W. Comparison of Beck Depression Inventories -IA and -II in psychiatric outpatients. J Pers Assess. 1996;67(3):588-597.

27. Spielberger C, Garsuch R, Lushane R. STAI: Manual for the State-Trait Anxiety Inventory. Palo Alto, CA: Consulting Psychologists Press; 1970.

28. Iikura Y. Treatment Guide for Obsessive-Compulsive Disorder. Osaka: Nihei-Sha; 1999.

29. Ashburner J. A fast diffeomorphic image registration algorithm. Neuroimage. 2007;38(1):95-113.

30. Genovese CR, Lazar NA, Nichols T. Thresholding of statistical maps in functional neuroimaging using the false discovery rate. Neuroimage. 2002;15(4):870-878.

31. Koprivova J, Horacek J, Tintera J, et al. Medial frontal and dorsal cortical morphometric abnormalities are related to obsessive-compulsive disorder. Neurosci Lett. 2009;464(1):62-66.

32. Pujol J, Soriano-Mas C, Alonso P, et al. Mapping structural brain alterations in obsessive-compulsive disorder. Arch Gen Psychiatry. 2004;61(7):720-730.

33. Togao O, Yoshiura T, Nakao T, et al. Regional gray and white matter volume abnormalities in obsessive-compulsive disorder: a voxel-based morphometry study. Psychiatry Res. 2010;184(1):29-37.

34. van den Heuvel OA, Remijnse PL, Mataix-Cols D, et al. The major symptom dimensions of obsessive-compulsive disorder are mediated by partially distinct neural systems. Brain. 2009;132(Pt 4):853-868.

35. Kim JJ, Lee MC, Kim J, et al. Grey matter abnormalities in obsessivecompulsive disorder: statistical parametric mapping of segmented magnetic resonance images. Br J Psychiatry. 2001;179:330-334.

36. Szeszko PR, MacMillan S, McMeniman M, et al. Brain structural abnormalities in psychotropic drug-naive pediatric patients with obsessivecompulsive disorder. Am J Psychiatry. 2004;161(6):1049-1056.

37. Atmaca M, Yildirim BH, Ozdemir BH, Aydin BA, Tezcan AE, Ozler AS. Volumetric MRI assessment of brain regions in patients with refractory obsessive-compulsive disorder. Prog Neuropsychopharmacol Biol Psychiatry. 2006;30(6):1051-1057.
38. Milad MR, Quinn BT, Pitman RK, Orr SP, Fischl B, Rauch SL. Thickness of ventromedial prefrontal cortex in humans is correlated with extinction memory. Proc Natl Acad Sci U S A. 2005;102(30):10706-10711.

39. Milad MR, Rauch SL. The orbitofrontal cortex and anxiety disorders. In: Zald DH, Rauch SL, editors. The Orbitofrontal Cortex. New York: Oxford University Press; 2006:523-543.

40. Huyser C, van den Heuvel OA, Wolters LH, et al.Increased orbital frontal gray matter volume after cognitive behavioural therapy in paediatric obsessive compulsive disorder. World J Biol Psychiatry. 2013;14(4):319-331.

41. Huyser C, van den Heuvel OA, Wolters L, et al. A longitudinal VBM study in paediatric obsessive-compulsive disorder at 2-year follow-up after cognitive behavioural therapy. World J Biol Psychiatry. 2014;15(6):443-452.

42. Gilbert AR, Mataix-Cols D, Almeida JR, et al. Brain structure and symptom dimension relationships in obsessive-compulsive disorder: a voxelbased morphometry study. J Affect Disord. 2008;109(1-2):117-126.

43. Cannistraro PA, Makris N, Howard JD, et al. A diffusion tensor imaging study of white matter in obsessive-compulsive disorder. Depress Anxiety. 2007;24(6):440-446.

44. Chiu CH, Lo YC, Tang HS, et al. White matter abnormalities of fronto-striato-thalamic circuitry in obsessive-compulsive disorder: a study using diffusion spectrum imaging tractography. Psychiatry Res. 2011;192(3):176-182.

45. Aouizerate B, Guehl D, Cuny E, et al. Pathophysiology of obsessive-compulsive disorder: a necessary link between phenomenology, neuropsychology, imagery and physiology. Prog Neurobiol. 2004;72(3):195-221.

46. Menzies L, Chamberlain SR, Laird AR, Thelen SM, Sahakian BJ, Bullmore ET. Integrating evidence from neuroimaging and neuropsychological studies of obsessive-compulsive disorder: the orbitofronto-striatal model revisited. Neurosci Biobehav Rev. 2008;32(3):525-549.

47. Carmona S, Bassas N, Rovira M, et al. Pediatric OCD structural brain deficits in conflict monitoring circuits: a voxel-based morphometry study. Neurosci Lett. 2007;421(3):218-223.

48. Gilbert AR, Moore GJ, Keshavan MS, et al. Decrease in thalamic volumes of pediatric patients with obsessive-compulsive disorder who are taking paroxetine. Arch Gen Psychiatry. 2000;57(5):449-456.

49. Szeszko PR, MacMillan S, McMeniman M, et al. Amygdala volume reductions in pediatric patients with obsessive-compulsive disorder treated with paroxetine: preliminary findings. Neuropsychopharmacology. 2004;29(4):826-832.

50. Mataix-Cols D, Marks IM, Greist JH, Kobak KA, Baer L. Obsessivecompulsive symptom dimensions as predictors of compliance with and response to behaviour therapy: results from a controlled trial. Psychother Psychosom. 2002;71(5):255-262.
Neuropsychiatric Disease and Treatment

\section{Publish your work in this journal}

Neuropsychiatric Disease and Treatment is an international, peerreviewed journal of clinical therapeutics and pharmacology focusing on concise rapid reporting of clinical or pre-clinical studies on a range of neuropsychiatric and neurological disorders. This journal is indexed on PubMed Central, the 'PsycINFO' database and CAS,

\section{Dovepress}

and is the official journal of The International Neuropsychiatric Association (INA). The manuscript management system is completely online and includes a very quick and fair peer-review system, which is all easy to use. Visit http://www.dovepress.com/testimonials.php to read real quotes from published authors. 